\title{
A System to Support Dissemination of Knowledge and Sharing of Experiences in the Working Environment \\ Review Code: 066
}

\author{
George Lepouras ${ }^{1}$, Costas Vassilakis ${ }^{1}$, George R. S. Weir ${ }^{2}$ \\ ${ }^{1}$ Department of Informatics and Telecommunications, University of Athens, 15784 \\ Athens, Greece \\ \{G.Lepouras, C.Vassilakis\}@di.uoa.gr \\ ${ }^{2}$ Department of Computer and Information Sciences, University of Strathclyde, \\ Glasgow G1 1XH, UK \\ gw@ cis.strath.ac.uk
}

\section{Dr George Lepouras}

Dr George Lepouras was born in Athens in 1967. He holds a B.Sc. in Mathematics (1991) from University of Athens, an MSc in Information Technology (1992) from University of Strathclyde, Scotland and a Ph.D. in Human-Computer Interaction (2000) from University of Athens. Dr. Lepouras has participated in several European and national RTD projects and has authored scientific papers in international conferences and journals and has also been a consultant to the General Secretariat for Information Systems of the Greek Ministry of Finance.

\section{Dr Costas Vassilakis}

Dr Costas Vassilakis was born in Arta, Greece in 1968. He holds a B.Sc. in Informatics (1990) from the Department of Informatics, University of Athens and a Ph.D. from the same department in 1995. Dr. Costas Vassilakis has participated in several European and national projects and has published scientific papers in international journals and conferences. He has been a Special Advisor for the General Secretariat for Information Systems of the Ministry of Finance, Greece (October 1998 - January 2002), and a one-year contract lecturer in the University of Athens.

\section{Dr George R.S. Weir}

George Weir is a lecturer in Computer Science at the University of Strathclyde, in Glasgow, Scotland where he teaches Human-Computer Interaction and Multimedia. His special interest lies in on-line education and aspects of user support. He has written and edited several books and papers in HCI. He has a doctorate in Philosophy from Edinburgh University (1983) and a Master of Arts from the University of Glasgow (1976). 


\section{Abstract}

In the information era enterprises strive to be productive and efficient. One feature of this goal is to engage their employees in education programmes, help them gain new experiences and knowledge and adapt to an ever-changing working environment. Such programmes require thorough design in order to achieve satisfactory results. Lately, enterprises recognising the role technology can play in the education of their employees, have adopted systems that supplement the traditional educational model with mechanisms that enable the sharing of experiences and knowledge [5]. In this paper we describe an architecture and a system prototype that allows users to search easily for information, interact with colleagues and share experiences, to compose and disseminate best practices and knowledge. The design of this system is based on insights gained from the operation of the Greek Taxation System.

\section{Keywords}

Life-long learning, organisational learning, support for creativity.

\section{Introduction}

Many modern enterprises recognise the productivity benefits from well-trained and educated personnel. The public sector has also adopted private sector approaches in order to secure well trained employees. Education programmes in the working environment help employees gain new experiences and knowledge and adapt better to an ever-changing working environment. Such programmes require thorough design in order to achieve satisfactory results and may have significant cost both in terms of financing and in terms of operational overheads.

As an example, the Greek Ministry of Finance spent over 2.5 million Euros in a twoyear timeframe, just for the training of employees, in aiming to achieve an improved level of service [7]. Other training activities also incurred considerable financial cost. Aside from the cost, such training programmes had an impact on the organisation's normal operation: during the educational programme, certain departments of the Tax Offices had to suspend or minimise their operation, while for some of the training activities employees had to move to other cities, where training was located.

Such a major training programme is required whenever a new system is installed or the number of accumulated revisions (usually due to legislative and organisational changes) renders past education obsolete. Changes occurring between such educational activities are not systematically communicated to employees and employees have to rely on informal and subjective methods in order to keep up to date.

Enterprises seek models and technologies that integrate the learning procedure into every day work, aiming to alleviate problems endemic in the traditional educational approach, such as lack of creativity and innovation, coping with change, insufficient support of school to work transition and inappropriate use of the 'gift wrapping approach' [6]. Organisational learning introduces a model where the organisation learns from its employees and subsequently makes this knowledge available to all employees. 
Shneiderman [10] presents a paradigm that identifies four key phases in the operation of any integrated framework for creativity support:

- Collect: learn from previous works stored in digital libraries, the web, etc.

- Relate: consult with peers and mentors at early, middle and late stages

- Create: explore, compose, discover and evaluate possible solutions

- Donate: disseminate the results and contribute to digital libraries, the web, etc.

Based on experience gained from work with the Greek Ministry of Finance, we propose a system that applies this paradigm, and supports each of its four key phases. The proposed system lays emphasis on the seamless integration of the organisational learning process into everyday work, thereby extending options available to the users in a coherent and intuitive manner.

In the remainder of this paper, section 2 provides an overview of the current state-ofthe-art, while section 3 describes the architectural design of the system and section 4 focuses on issues related to system design and implementation - including information on the current system prototype. Section 5 presents our conclusions and, finally, section 6 outlines our plans for future work.

\section{Alternative Approaches}

Fischer et al. [5], present a system where users can collaborate with each other by sharing and updating group memories. This system helps project teams to capture, store, organize, share, and retrieve electronic mail conversations. A central information space stores mail communication of all groups, categorised by group and subject, and a number of methods is afforded for group members to retrieve information from this repository (browsing in reverse chronological order, browsing according to project-specific categories, free text queries and information delivery of related mail messages).

The CEDAR approach [11] attempts to formulate training practices that move from a one-shot training process to continuous learning and shift the training focus from the individual to the group and organisation level. In order to achieve these goals, CEDAR employs (a) a document-centred discourse space for structuring discussions about representations of work, (b) client tools for articulation and refinement of domain concepts, (c) mechanisms for knowledge delivery and access that support individual and group learning, and (d) an organisational memory server for knowledge capture, structuring and maintenance. The main tool available to CEDAR users is the team workbook, a web-based environment through which teams articulate, discuss and review their plans, share experiences and best practices and augment corporate knowledge.

The ENRICH methodology [9] incorporates theories of learning at individual, group and organisational level, interweaving working and learning. This is based on a formal ontology and a model for managing work representations and best practices. The ontology is able to capture process, domain and media knowledge, and each of the roles identified by the methodology (team member, best practice coordinator, 
local developer) is equipped with a set of web tools to enrich work products with context information. The tools support collaborative learning by integrating html documents, discussion forums/threads and knowledge models.

Bergenti et al [1] exploit agent technology to provide a system that facilitates storage and retrieval of information from a corporate knowledge base. The system includes five categories of agents: personal assistant agent to support searching and storing, user profile agent to manage user preferences, document ontology to annotate documents, search agent, and archiving agent.

Cerbah and Euzenat [2] identify an important dimension of organisational learning, namely terminological extraction, which is combined with document indexing. This enriched document indexing component is integrated through XML interfaces with a knowledge management and model indexing component to formulate a system that supports the creation and maintenance of knowledge repositories. Users interact with the system through XTerm, a natural language processing system that facilitates terminology acquisition from documents and terminology-centred hypertext navigation. Furthermore, users are assisted in the process of creating links between informal information sources and formal models.

We might also note that the value of an organisational learning process is lately recognised by the software industry, and commercial platforms for supporting this process are emerging. For instance, the Aspen learning experience server [3] provides an environment where users can take on-line courses, customise their learning experience and share their knowledge with other users. Enterprises installing the Aspen server appoint an administrator, for managing courses and users, while the task of knowledge creation is assigned to designated experts.

In some of the approaches presented above, the relationship between knowledge management and online lifelong learning is explicitly identified, whereas in other approaches this relationship is implied. In [4] the benefits from integrating knowledge management and online learning are summarised and tactics and tools for knowledge management and online learning are described.

Notably, while substantial work has been done in the areas of content management, formal models, process models and overall functionality for organisational memory systems, the user interface aspect of these systems has received scant attention. Shneiderman's work [10] is a noteworthy exception, aiming to support creativity through information visualisation. Still, in all cases, users access the facilities of the online learning system through an application that is distinct from the applications that they usually employ in their normal life of work; moreover, the interfaces provided to the users for accessing the online learning system, although usually adequate, contain only the elements that are absolutely necessary for the accomplishment of the tasks provided by the system. These two facts tend to inhibit users from getting the most out of the system, and in some cases users refrain from using it. In this paper, we propose an integrated environment for work and online learning that removes the burden of application switching and multiple work environments. Additionally, this environment offers a rich interface that, together with the back-end servers, provides easy access to corporate knowledge. 


\section{Architectural Design}

As noted in section 1, the proposed system seamlessly integrates the phases of collection, relation, creation and donation of information, into every day work. Employees may use the system to search for information on a specific issue, interact with other employees, discuss solutions and practices, compose or update information and disseminate this information to their peers. The system delivers this functionality through a number of subsystems: databases of previous questions, annotations and best practices, a subsystem for the processing of information and knowledge extraction, and - in principle - connection to external databases.

When users $\log$ in to the system for the first time they are prompted to create a personal profile. The profile contains information about the user that can assist the system in characterising specific information as of interest to a user. The user profile may contain information regarding the user's department and job responsibilities, and user's personal preferences for information type (laws, explanatory comments, best practices, etc.).

In the context of the Greek Ministry of Finance, the collection phase is supported by facilities to search for legislature-related information, best practices, examples, and expert opinions. Users are not required to search these databases separately: a concept query is submitted to the correlation server, which retrieves all appropriate information from the available databases. The correlation server then inserts semantic links between the retrieved items of information to create a semantically rich document, which is returned as a response to the query.

An emergent problem with queries was that users often expressed the same concept using different words or abbreviations. For instance, the concept 'VAT' might also be referred to as 'Value Added Tax'. The initial version of the query engine did not take this issue into account. Consequently, results returned to the users were often partial. To accommodate this issue, the query engine was supplemented with a terminology dictionary, which provides equivalencies between homonyms, in a manner similar to the semantics of the "has-synonyms" slot described in [13]. In our case, however, some terms have varying meanings in different contexts (e.g. the term tariff has different interpretations in the contexts of customs and tobacco sales). For this reason, the terminology dictionary adopts a hierarchical structure, with the top level providing context, and the second level expressing term equivalencies within this context.

For the relation phase, the system enables users to query other employees. The query may be submitted to a specific person or be broadcast to a selected group of employees. Besides free text, the query may contain a range of structured elements, such as thematic categories (e.g. VAT, income tax etc.), interest groups, or keywords, to assist the message recipients to classify and handle it more efficiently. These structured elements are stored by the system and exploited in the information retrieval and correlation process.

The creation phase is facilitated by tools that help users explore approaches other employees have taken, input their annotations for their own or for public use, compose new responses to problems faced, etc. Finally, the dissemination phase can be supported by making all of this information available to other system users. Knowledge is donated either explicitly, by filling in the suitable forms, or implicitly, 
by replying to a question submitted by another user. In all cases, the donor may input a degree of confidence, which is then available to readers of this piece of information.

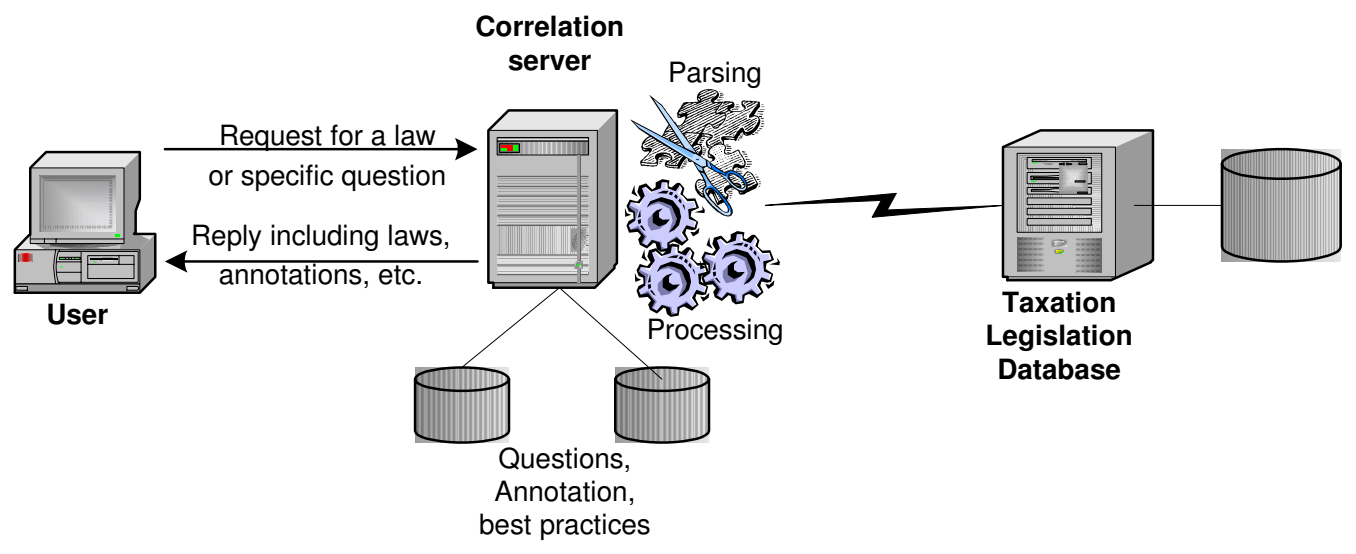

Figure 1: System Architecture

The architectural design of this system is illustrated in Figure 1. This is derived in part from previous work [8], [12] and has been enhanced to allow for the extra user support needed for the realisation of the knowledge-sharing paradigm.

\section{Design Issues}

The main goal in the creation of an effective user interface for this system is to enable transparent knowledge acquisition and easy exploration of the organisation's knowledge base. Three obstacles may hinder this process: lack of content, lack of useful retrieval functions and inaccurate information. In the Ministry of Finance case study, availability of basic content is ensured by the presence of the taxation legislation database. This database contains laws, legislation, resolutions and interpretation clauses. The database is kept up-to-date by a group of law experts.

However, the presence of the legislation database alone is not enough to guarantee that the system contains the type of information that will secure its adoption by end users. A user may seek information on a particular case that is not addressed directly by a specific law or which may fall under more than one law. To this end, it is necessary that tacit or implicit knowledge also be recorded. Tacit knowledge is probably more powerful than explicit knowledge, e.g. the awareness of a certain law or a directive, but as it is drawn from experience it is also more difficult to express formally and share it with colleagues. Furthermore, since such knowledge imbues a type of power, people may often be reluctant to share it.

To facilitate the collection of information, the system should offer the possibility of a full text search against the knowledge base. This capability can be combined with a multilingual keyword search. Although the legislative database caters for most cases, for some cases (especially those concerning cross-border transactions, within the European Union) the user may need to search a database of European directives. In such instances, a user need not be acquainted with the terminology in other languages. The system should enable multilingual searches, whereby the user may enter keywords in the local language and retrieve corresponding legislative information in any language. 
In contrast with existing systems, the proposed approach offers the possibility for invalidation of knowledge. Since procedures and best practices often rely on related laws or directives, once a law is abolished or superseded by new legislation, the system can locate all information based on the obsolete law and characterise it as 'outdated'. Information will remain in the system, but any user trying to retrieve such information will be appraised that a new law has come into force that affects this information. Even though the system could delete this information, such functionality was deemed inappropriate since in some (older) cases the previous law may take effect, or may be used to explain previously elected decisions.

The relaying of information to peers or senior colleagues can be carried out through an integrated mail program. This program provides all standard email functionality along with some features that can help users to collaborate with less effort. Apart from being able to send email to anyone whose username is known, users can also send email to peers who share the same position or job function within the organisation, or, indeed, anyone whose profile matches specific interests and who has selected to accept incoming mail from a non-authorised person. The latter strategy was deemed appropriate to minimise the intrusion of mail broadcasting in large organisations.

To facilitate the creation of new knowledge, the system offers a composing function. Users can copy information they find interesting, link pieces of information together by hyperlinks or file information. These processes can be carried out automatically or manually. For example, when a user composes a new document the system can automatically propose adding a link when it identifies a keyword (such as Law 5055/1994). The system may also offer to categorise the new information (and emails) by means of keywords in the text or user's profile. This type of functionality enables the user to focus on their job rather than trying to learn how to master the system.

To this end, if the author designates it, the system can automatically make new pieces of information available to other users. Depending on profile settings, users can select to be notified when new information becomes available on topics of their interest.

The next two images illustrate the interface for the search facility ${ }^{1}$.

\footnotetext{
${ }^{1}$ Text relating to commands has been translated into English, in order to be meaningful to an international audience.
} 


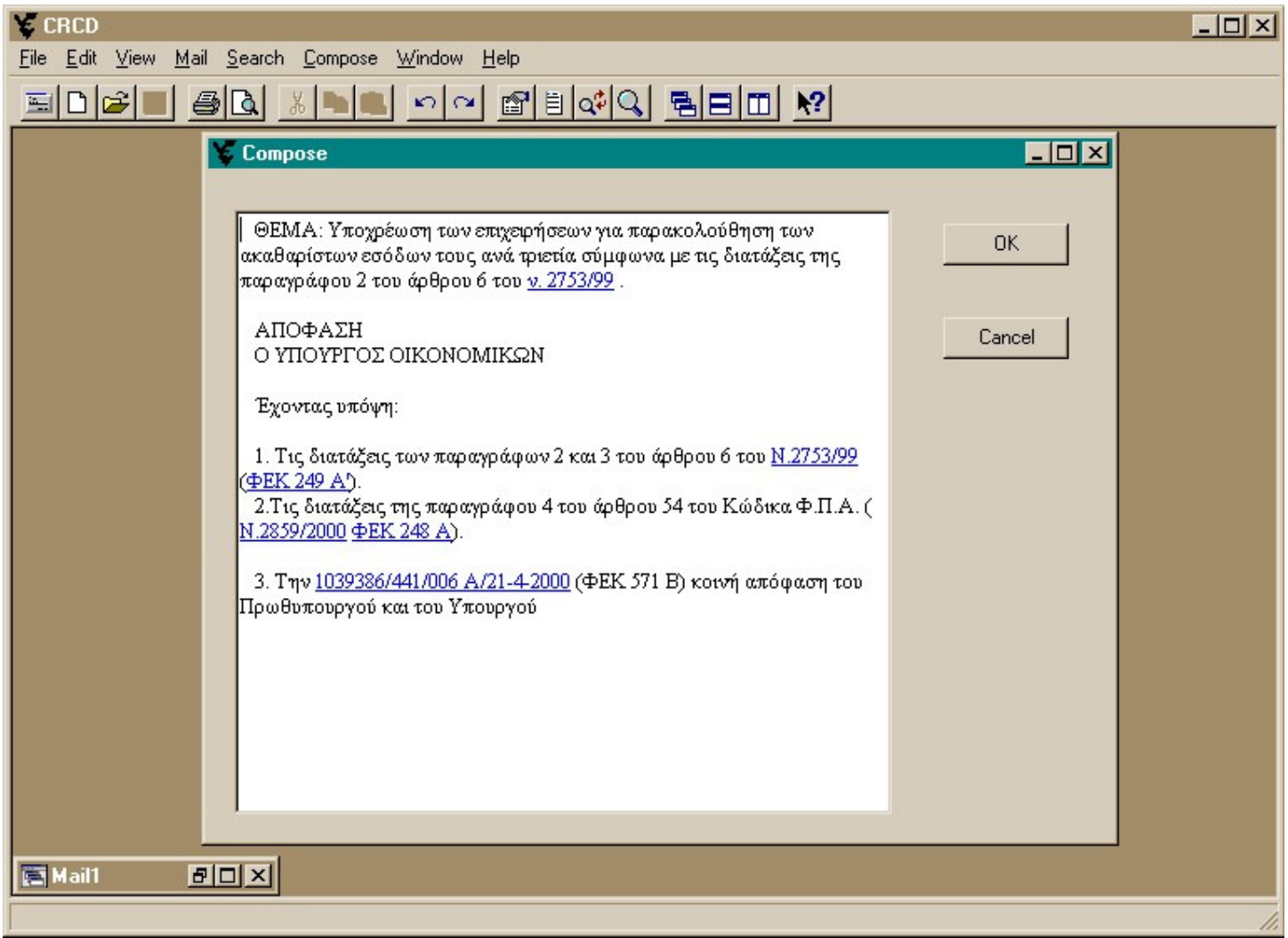

Figure 2: Composer Window

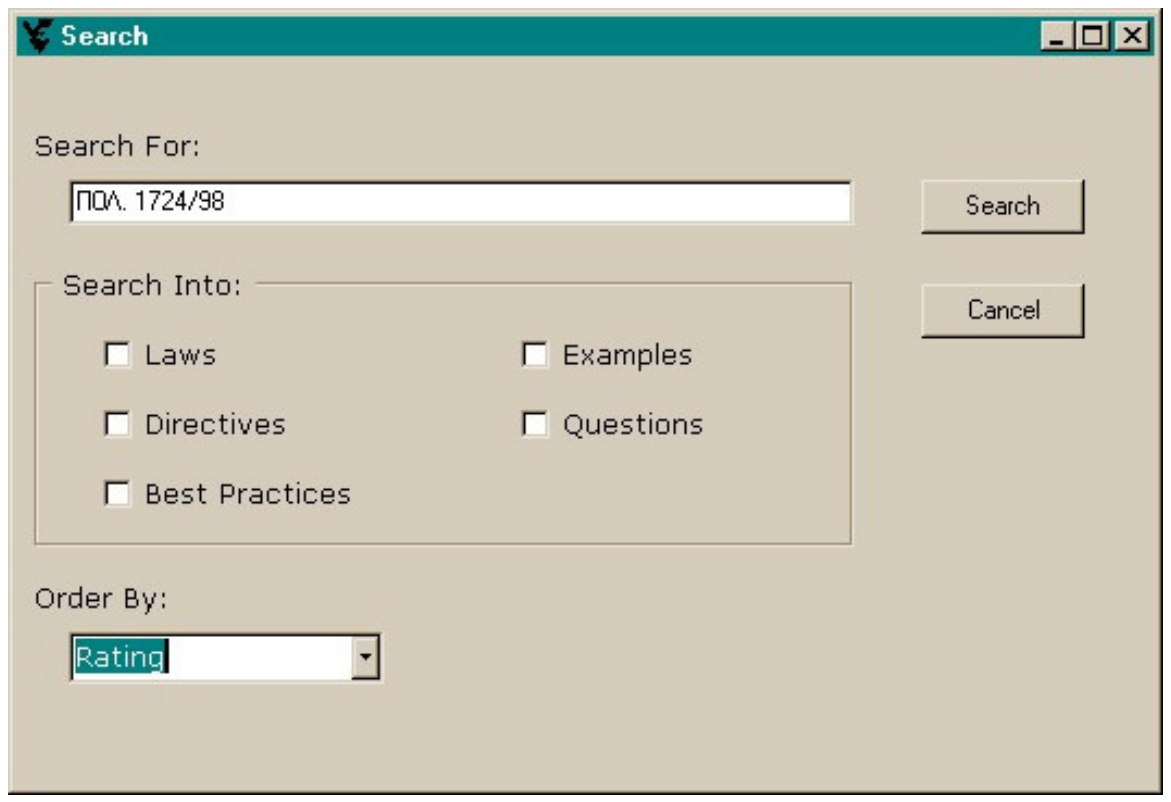

Figure 3: Search Dialog Box

The system has to support the transparent acquisition of knowledge. This is secured through the integrated environment, wherein users can exchange email with colleagues, search for legislative information and kept up to date with information regarding their interests. Since the system incorporates the email messenger it can easily and transparently categorise messages according to its terminology database. 
Users are able to enter their own comments, annotations or questions into the system. At all times, the system should support transparent knowledge acquisition with minimum intrusion in users' everyday activities.

\section{Discussion and Conclusions}

Employee education is regarded by many organisations as a task distinct from every day work. This approach jeopardises knowledge created in the ordinary line of work, leaving it to individuals. In such circumstances, this knowledge is poorly exploited. The approach recommended in the present paper, provides mechanisms for information retrieval, creation and dissemination. Additionally, disseminated information is transparently stored in an organisational repository, making it available not only for current users of the system, but newcomers as well.

A primary goal in our system design was the seamless integration of information retrieval, creation and dissemination mechanisms into everyday work activities. Using two distinct systems, one for knowledge management and one for the normal line of work, will discourage use of the former, since, even in windowing environments that support concurrent tasks and task switching, users refrain from using more than one application at any given time. With this in mind, each work screen was enriched with appropriate, context-sensitive user interface widgets providing access to additional system functionality. After a short presentation on the functionality of the new widgets, most users were able to use its novel features without significant problems. Since most users were already accustomed to navigating between hyperlinked documents, adoption of a web-style paradigm for presenting the ancillary content facilitated easy user adoption of the system.

We believe that the advocated system can considerably improve the education of users in their working environment.

\section{Future Plans}

Currently, a system prototype has been developed and is being evaluated in selected user groups with encouraging results. Future plans include interfacing the system to external knowledge databases (e.g. court decisions) and adding a computer-based training module, for delivering 'packaged' knowledge. The computer-based training module is expected to prove particularly useful for new employees, who need to receive 'batch' training, and for disseminating organised knowledge to experienced users.

\section{References}

[1] Bergenti F., Poggi A. and Rimassa G. (2000) Agent Architecture and Interaction Protocols for Corporate Memory Management Systems, Proceedings of the ECAI Workshop on Knowledge Management and Organisational Memories, Berlin.

[2] Cerbah F. and Euzenat J. (2000) Integrating Textual Knowledge and Formal Knowledge for Inproving Tracability, Proceedings of the ECAI Workshop on Knowledge Management and Organisational Memories, Berlin. 
[3] Click2Learn Corporation Aspen Learning Experience Server. Retrieved from $\mathrm{http} / / /$ home.click2learn.com/en/aspen/aspen_lxs.asp

[4] The Conference Board, Beyond Knowledge Management: New ways to Work and Learn (Research Report 1262-00-RR). Retrieved December 9, 2001, from http://www.sveiby.com.au/articles/confboard.pdf

[5] Fischer G., Lindstaedt S., Ostwald J., Schneider K. and Smith J. (1996). Informing System Design Through Organizational Learning, Proceedings of the Second International Conference on The Learning Sciences, Northwestern University, Evanston, Association.

[6] Fischer G. (2000). Lifelong Learning And Its Support With New Media. In N.J. Smelser and P. B. Baltes (Ed.): International Encyclopedia of Social and Behavioral Sciences, (in press) Elsevier. Retrieved from http://www.cs.colorado.edu/ gerhard/papers/iesbs2001.pdf.

[7] Final Report on the Activities of the Business Programme "Kleisthenis" (n.d.). Retrieved November 25, 2001, from General Secretariat for Information Systems of the Greek Ministry of Finance, http://www.gsis.gov.gr/04/kleisth/

[8] G. Lepouras, C. Vassilakis, G.R.S. Weir (2002) Serving Enhanced Hypermedia Information. In F. Crestani, M. Girolami, and C.J. van Rijsbergen (Ed.) Lecture Notes in Computer Science, ECIR 2002, (pp. 86-92). Springer.

[9] Mulholland P., Dominigue J., Zdrahal Z. and Hatala M. (2000). Supporting Organisational Learning: an Overview of the ENRICH approach, Journal of Information Sciences and Use, 20(1), 9-23.

[10] Shneiderman B. (1999). Supporting Creativity with Advanced InformationAbundant User Interfaces", HCIL Technical Report No. 99-16. Retrieved from http://www.cs.umd.edu/hcil

[11] Sumner T., Dominigue J., Zdrahal Z., Millican A. and Murray J. (1999). Moving from On-the-Job Training towards Organisational Learning, Proceedings of the 12th Banff Knowledge Acquisition Workshop, Banff, Alberta, Canada.

[12] Weir, G.R.S. and Lepouras, G. (2001). English Assistant: A Support Strategy for On-Line Second Language Learning, ICALT 2001, IEEE International Conference on Advanced Learning Technologies, Madison, USA.

[13] Zdrahal Z., Mulholland P., Dominigue J., and Hatala M. (2000). Sharing Engineering Design Knowledge in a Distributed Environment, Journal of Behaviour and Information Technology, 19(3), 189-200. 